Hagen Frank*, Ariane Dierke, Christoph Brandt-Wunderlich, Eric Bohne, Thomas Kuske, Michael Stiehm, Klaus-Peter Schmitz and Stefan Siewert

\title{
Numerical design studies of meander structures for self-expanding absorbable stents
}

\begin{abstract}
Absorbable stents made of poly-L-lactic acid (PLLA) exhibit inferior mechanical properties compared with metal based devices. Therefore, design optimization by means of finite element analysis (FEA) plays an important role in development of high performance absorbable stents. In the present study, three different meander structures made of PLLA material were compared in terms of their radial resistive force (RRF) using FEA. Material tests were performed to obtain input parameters of PLLA for the simulation. FEA was carried out to simulate the RRF and the length change of the meander structures as a function of the diameter. In addition, the numerical results were compared to the experimental outcome using corresponding meander based stents performing radial testing with a segmented head mechanism. Comparable high stresses occurred in the Ubends of the examined stent designs. In designs with a large radius of curvature less stress was observed while crimping. One meander structure developed comparatively higher structural resistance against radially acting forces, due to its curved strut design. In general, FEA revealed consistent results compared to the experimental investigations.
\end{abstract}

Keywords: Finite-element analysis, self-expanding stents, polymeric stent, PLLA, meander design study.

https://doi.org/10.1515/cdbme-2021-2188

\section{Introduction}

Absorbable self-expandable stents made of poly(L-lactic acid) (PLLA) represent an alternative to permanent metallic devices. Self-expandable stents have in general a greater flexibility than balloon-expandable stents. This predestines them for use in strongly tortuous or difficult-to-reach vessels. Biodegradation of the stent material offers the substantial advantage to use antiplatelet therapy of patients only temporarily, subsequent to an endovascular therapy. Furthermore, re-interventions become possible. This decisively increases the quality of life, especially for young patients, e.g. receiving treatment in gynecological or any endovascular therapy, respectively [1].

Due to inferior material properties of PLLA in comparison to metallic stent materials, design optimization by means of finite element analysis (FEA) is of even greater importance in development of absorbable stents. Meander dimensions were chosen in consideration of common vascular vessel geometry stated in literature [2]. Aim of the study was to assess the impact of design elements radius of curvature and curved struts on the substructure of a meander under radial loading.

\section{Materials and Methods}

\subsection{Design of the stent structures}

Three different meander structures were designed using the computer-aided design (CAD) software package Creo Parametric 6.0 (Parametric Technology Corp., Needham, MA, USA). The corresponding stent structures consist of eight cells around the circumference and were designed with an inner diameter of $4 \mathrm{~mm}$, a wall thickness of $100 \mu \mathrm{m}$ and a strut width of $100 \mu \mathrm{m}$. In the U-bends, different radii of curvature were applied (see Figure 1). The radius of curvature in the struts of design $\mathrm{C}$ was set at $1.35 \mathrm{~mm}$.
*Corresponding author: Hagen Frank: Institute for ImplantTechnology and Biomaterials e.V., Rostock-Warnemünde, Germany, E-Mail: hagen.frank@uni-rostock.de Ariane Dierke, Christoph Brandt-Wunderlich, Eric Bohne, Thomas Kuske, Michael Stiehm, Klaus-Peter Schmitz, Stefan Siewert: Institute for ImplantTechnology and Biomaterials e.V., RostockWarnemünde, Germany

Klaus-Peter Schmitz: Institute for Biomedical Engineering, Rostock University Medical Center, Rostock-Warnemünde, Germany, 


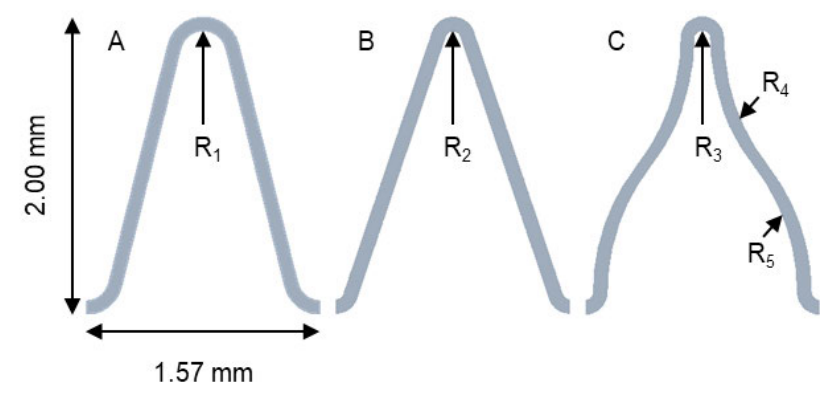

Figure 1: Design $A$ to $C$ with total dimensions of the numerical and experimental examined meander design structures $\left(R_{1}=150 \mu \mathrm{m}, R_{2}=R_{3}=50 \mu \mathrm{m}\right.$ and $\left.R_{4}=R_{5}=1.35 \mathrm{~mm}\right)$.

\subsection{Developing a material model}

Uniaxial tensile tests were conducted to determine the material parameters of PLLA for FEA using test specimen according to DIN EN ISO 527-2 (type 1BB) [3]. The test samples were manufactured from dip coated semi-finished products and shaped by femtosecond laser cutting $(n=10)$. Due to scattering of the individual measurements an averaging was performed. Figure 2 shows the resulting stress-strain curve.

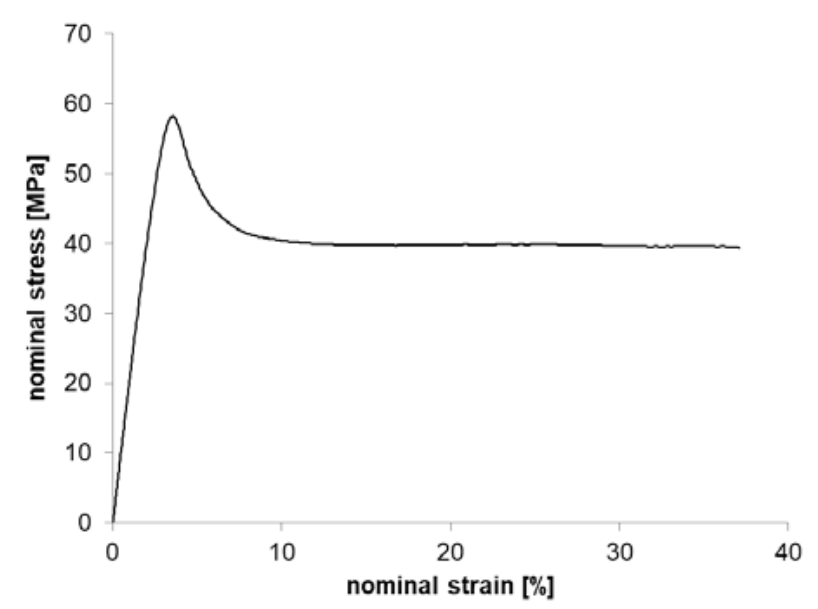

Figure 2: Averaged nominal stress-strain curve of PLLA test specimens $(n=10)$ measured at a test temperature of $37^{\circ} \mathrm{C}$.

A generalized isotropic model with an elastic and plastic proportion was defined. The elastic model was defined by a Young's modulus of 2,073 $\mathrm{N} \mathrm{mm}^{-2}$ obtained from material testing. The used Poisson's ratio of 0.45 was chosen from literature [4]. To describe the plasticity of the model, material test data was converted for FEA input.

\subsection{Numerical simulation}

FEA was performed using Abaqus/Standard 2021 (Dassault Systèmes, Vélizy-Villa-coublay, France). The simulated load case was a crimping process of the meander structures from fully expanded state down to a final diameter of $1.5 \mathrm{~mm}$. Therefore, the nodes of a cylindrical shell surface (crimping tool) were applied with boundary conditions to realize the desired radial displacement. The meander structures were constraint in tangential direction on both sides of the structure.

A surface-to-surface contact was applied, defining the outer surface of the meander structure as slave and the inner surface of the crimping tool as master surface using a frictionless tangential behaviour and the hard contact pressure-overclosure relationship. Augmented Lagrange constrain enforcement method was used to model the normal contact behaviour. For stable convergence a standard contact control was implemented into the FEA model to allow a surface penetration of $10 \mu \mathrm{m}$. Meshing parameters are given in Table 1.

The radial resistive force (RRF) of the meander structures was obtained by the summation of all nodal reaction forces in radial direction of the crimping tool.

Table 1: Overview of the used meshing parameters.

\begin{tabular}{lll}
\hline & $\begin{array}{l}\text { meander } \\
\text { structure }\end{array}$ & $\begin{array}{l}\text { crimping } \\
\text { tool }\end{array}$ \\
\hline elements & solid & shell \\
element type & C3D8 & SFM3D4 \\
global edge length & $0.04 \mathrm{~mm}$ & $0.1 \mathrm{~mm}$ \\
\hline
\end{tabular}

\subsection{Experimental radial testing}

For experimental radial testing three corresponding $20 \mathrm{~mm}$ meander based stents with similar connectors per design were used.

Measurements of the RRF were conducted using a segmented head testing machine (Blockwise, Tempe, USA) from the expanded state to a minimum diameter of $1.5 \mathrm{~mm}$. Measurements were carried out at a cross head speed of $0.05 \mathrm{~mm} \mathrm{~min}^{-1}$ at $37^{\circ} \mathrm{C}$. Testing was in accordance to ASTM F3067-14 [5]. 


\section{Results}

FEA revealed maximum stresses in the U-bends of the designs. Up to 21.6 MPa less maximum stress (Mises) was observed in design A with a large radius of curvature. Due to its curved strut design the meander structure $\mathrm{C}$ develops a spring link behavior. As a result reduction of plastic strain was determined, leading to comparatively high structural resistance against radially acting forces. Table 2 states the observed characteristic performances of the examined structures.

Table 2: Structural performance in terms of maximum length change, plastic strain and stress by design.

\begin{tabular}{|c|c|c|c|}
\hline & Design A & Design B & Design C \\
\hline $\begin{array}{l}\text { Maximum length } \\
\text { change }[\mu \mathrm{m}]\end{array}$ & 2.2 & 2.6 & 8.1 \\
\hline $\begin{array}{l}\text { Equivalent plastic } \\
\text { strain [\%] }\end{array}$ & 2.21 & 3.50 & 2.52 \\
\hline $\begin{array}{l}\text { Maximum stress } \\
\text { (Mises) [MPa] }\end{array}$ & 51.5 & 73.1 & 57.8 \\
\hline
\end{tabular}

The greatest length change, the lowest plastic strain and medium stress was observed in Design C (see Figure 3).

Additionally, the meander length change was calculated as a function of the diameter for each individual design (see Figure 4).
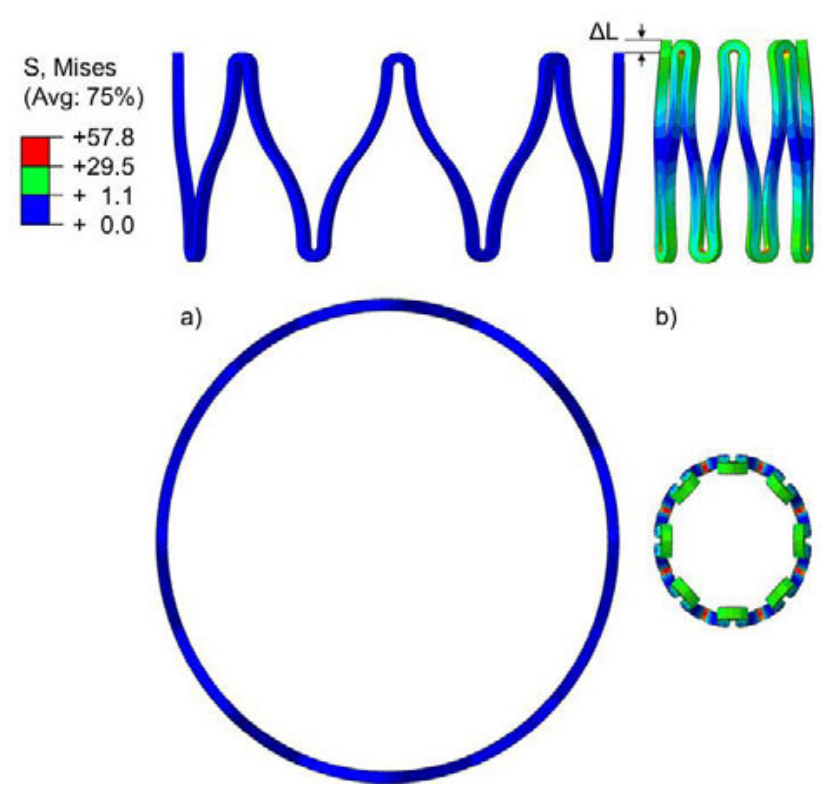

Figure 3: Meander structure $C$ in side view (top) and top view (bottom): (a) Initially expanded state with $4.2 \mathrm{~mm}$ outer diameter, (b) final crimped state with $1.5 \mathrm{~mm}$ outer diameter and lengthening $\Delta L=8.1 \mu \mathrm{m}$.

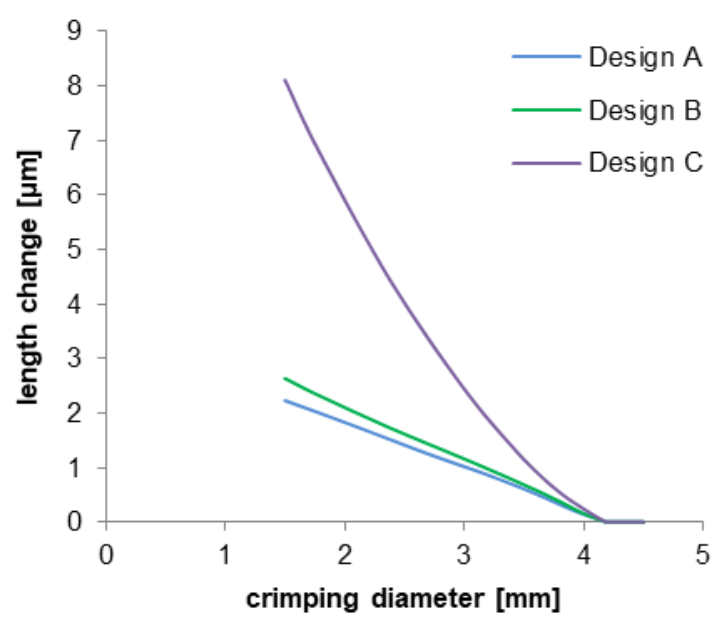

Figure 4: Computed structural length change by design.

Comparing the obtained experimental and numerical RRFs differences in the results were observed. Nevertheless, consistency throughout the results of each design was seen. To compensate for the ideal behaviour of the numerical model an offset of $0.45 \mathrm{~N}$ for the numerical values was introduced (see Table 3).

Table 3: Numerical RRF including an offset of $0.45 \mathrm{~N}$ and experimentally obtained RRF compared by design at $4,3,2$ and $1.5 \mathrm{~mm}$ crimping diameter.

\begin{tabular}{|c|c|c|c|c|c|}
\hline \multirow[t]{2}{*}{ Design } & \multirow[t]{2}{*}{ Method } & \multicolumn{4}{|c|}{ RRF [N] for various crimping diameters } \\
\hline & & $4 \mathrm{~mm}$ & $3 \mathrm{~mm}$ & $2 \mathrm{~mm}$ & $1.5 \mathrm{~mm}$ \\
\hline \multirow[t]{2}{*}{ A } & FEA & 0.50 & 0.67 & 0.81 & 0.88 \\
\hline & Experiment & 0.52 & 0.89 & 1.22 & 1.81 \\
\hline \multirow[t]{2}{*}{ B } & FEA & 0.52 & 0.70 & 0.89 & 0.98 \\
\hline & Experiment & 0.70 & 0.96 & 1.17 & 1.40 \\
\hline \multirow[t]{2}{*}{ C } & FEA & 0.51 & 0.67 & 0.80 & 0.86 \\
\hline & Experiment & 0.61 & 0.96 & 1.26 & 1.81 \\
\hline
\end{tabular}

\section{Discussion}

Within this study an elastic-plastic material model for PLLA was developed to assess three different meander designs by simulating a crimping load case. Consistent results between numerical and experimental outcome were determined. The study has shown that in terms of plastic strain radii of curvature in struts are preferable for the crimping load case. This design feature allows reducing radii in U-bends by twothirds obtaining similar plastic strain and also enabling smaller maximum crimp diameters. 
Design $\mathrm{C}$ was considered as most favourable. Due to its comparatively low plastic strain and medium stress value. Its length change was the largest but is of minor importance since it corresponds to a relative length change smaller than $0.5 \%$. This design is viewed as a great starting point for further design studies analysing other challenging load cases. A similar meander design was found in a biodegradable microstent for minimally invasive treatment of Fallopian tube occlusions, proving the outcome of this study [1].

Possible reasons for the deviation seen in the numerically and experimentally obtained RRF results are inaccuracies in the manufacturing process, leading to material defects and variations in the sample geometries. Also the determination of the change in length in the tensile test could be further improved by using larger tensile specimens. In general, however, the results of the tensile test were in good agreement with the data found in literature [6].

Further investigations should focus on developing an improved material model and its application for further design studies of polymeric self-expanding resorbable stent structures. The influence of changing geometry parameters on the agreement between experiment and numeric should also be investigated. Additionally another design study of connectors was considered important especially for bending load cases. For this purpose an experimental comparison or validation should also to be aimed at.

\section{Author Statement}

Research funding: Financial support by the Federal Ministry of Education and Research within RESPONSE "Partnership for Innovation in Implant Technology" is gratefully acknowledged.

Conflict of interest: Authors state no conflict of interest. Acknowledgement: Support of D. Passler is gratefully acknowledged.

\section{References}

[1] A. Dierke, F. Borowski, S. Großmann, C. Brandt-Wunderlich, C. Matschegewski, P. Rosam, N. Pilz, P. Reister, R. Einenkel, U. Hinze, J. Keiler, M. Stiehm, K. Schümann, A. Bock, Boris Chichkov, N. Grabow, A. Wree, M. Zygmunt, K.P. Schmitz and S. Siewert: Development of a biodegradable microstent for minimally invasive treatment of Fallopian tube occlusions. Current Directions in Biomedical Engineering. 2020;6(3):20203019

[2] Kretschmer T, Spetzler RF, Heros RC, Hernesniemi, J.: Zerebrale Aneurysmen und Gefässmalformationen. Behandlungsgrundlagen und neurochirurgische Therapie in Fallbeispielen. Berlin, Germany: Springer, 2017.

[3] ISO 527-2: Tensile Testing for Plastics, International Organization for Standardization, 2012.

[4] Schultze C (2010) Bioabsorbierbare koronare Polymerstents - Strukturmechanische und experimentelle Untersuchungen. http://d-nb.info/1014212103/34. Accessed 07 July 2021

[5] ASTM F3067-14. Guide for Radial Loading of Balloon Expandable and Self Expanding Vascular Stents.

[6] Engelberg I and Kohn J: Physico-mechanical properties of degradable polymers used in medical applications: a comparative study. In: Biomaterials, 1991. 12(3): 292-304 